04

\title{
Магнитоплазменный ускоритель макротел с массивным легкоплазменным инициатором
}

\author{
(C) А.В. Козлов, А.В. Котов, В.П. Полищук, А.В. Шурупов \\ Объединенный институт высоких температур РАН, \\ 125412 Москва, Россия \\ e-mail: polistchook@mail.ru
}

Поступило в Редакцию 6 февраля 2019 г.

В окончательной редакции 6 февраля 2019 г.

Принято к публикации 27 февраля 2019 г.

Представлены результаты экспериментов с инициатором из лития и графита, масса которых составляла $\sim 10 \%$ от массы ударника. Подобные инициаторы создают плазму с высоким значением скорости звука и обеспечивают начальную скорость ударника $\sim 0.5 \mathrm{~km} / \mathrm{s}$. Описаны каплеобразные повреждения на изоляционной стенке канала ускорителя. Отмечена связь между появлением этих повреждений и резким торможением плазменного поршня. Обсуждена гипотеза, связывающая возникновение повреждений с резонансной деформацией канала под действием движущегося фронта высокого давления. Показано, что гипотеза о резонансной деформации канала на качественном уровне объясняет известные особенности работы ускорителя.

Ключевые слова: рельсотрон, скорость звука, резонансная деформация.

DOI: $10.21883 / J T F .2019 .09 .48063 .37-19$

\section{Введение}

В магнитоплазменном ускорителе (МПУ) или рельсотроне метаемое тело (МТ) разгоняется под действием давления плазмы, расширяющейся в магнитном поле. Долгое время считалось, что МПУ смогут разогнать граммовые тела до скорости um свыше $10 \mathrm{~km} / \mathrm{s}$. В обзоре [1] приведены проекты МПУ, разрабатывавшиеся в США в конце прошлого века, в соответствие с которыми должны были быть получены скорости метания $20-50 \mathrm{~km} / \mathrm{s}$. Однако реальная скорость $u_{m}$ оказалась значительно меньше ожидаемой - „рекордные“ скорости МТ не превысили $7 \mathrm{~km} / \mathrm{s}$ [1].

Расхождение между фактическими и предполагаемыми значениями скоростей метания объясняют, в первую очередь, увеличением массы толкающей плазмы вследствие термического разрушения стенок канала и возникновения паразитных токовых структур в следе плазменного поршня (ПП) [1,2]. Были опробованы различные технологические решения для преодоления этих факторов, однако скорость метания практически не увеличилась. Это определило значительное снижение интереса к МПУ в последнее время.

В работах [3-5] рассматривалась гипотеза об ограничении скорости метания значением скорости звука cs в плазме МПУ. Скорость звука $c_{s}$ близка к тепловой скорости тяжелых частиц и поэтому зависит, главным образом, от температуры плазмы и ее состава или, огрубляя, от среднего молекулярного веса частиц плазмы $\mu_{m}$. Состав плазмы в канале известных конструкций рельсотронов определяется в основном продуктами разрушения его стенок. Скорость звука может быть, в принципе, увеличена до $10-12 \mathrm{~km} / \mathrm{s}$ при изготовлении стенок канала из материалов с низким молекулярным весом или нанесении соответствующих покрытий. В соответствии с гипотезой [3-5] тогда можно ожидать соответствующего увеличения скорости метания. В этом случае МПУ по своим возможностям заметно превзойдут двухступенчатые легкогазовые пушки, в которых максимальная скорость МТ составляет около $7 \mathrm{~km} / \mathrm{s}$ [6].

Наиболее простой путь увеличения скорости звука на начальном участке ускорителя - это использование массивного инициатора из проводника с низким молекулярным весом. Основная цель настоящей работы состояла в том, чтобы исследовать характеристики МПУ с подобными инициаторами из лития или графита.

\section{1. Влияние скорости звука на скорость метания}

Предположение, высказанное в работах [3-5], о том, что скорость метания в МПУ ограничена значением скорости звука $c_{s}$, к настоящему времени не доказано, и поэтому может рассматриваться в качестве правдоподобной рабочей гипотезы. Вместе с тем, поскольку в работе [7] она была подставлена под сомнение, целесообразно привести некоторые пояснения. Гипотеза [3-5] обосновывалась известными трудностями получения сверхзвукового течения в канале постоянного сечения [8], при этом теоретический анализ для условий канала МПУ в работах [3-5] не был представлен. Чтобы восполнить этот пробел, рассмотрим стационарное течение плазмы с плотностью $\rho$ и давлением $p$ в канале заданного сечения $A(x)$, где $x$ - продольная координата. В пренебрежении вязкостью и теплопроводностью течение описывается законами сохранения [9]:

$$
\operatorname{div}(\rho u)=g(x)
$$




$$
\begin{gathered}
\rho u \frac{d u}{d x}+\frac{d p}{d x}=f(x), \\
\operatorname{div}\left(\rho u\left(\varepsilon+\frac{u^{2}}{2}\right)+p u\right)=q(x) .
\end{gathered}
$$

Здесь $u-$ скорость потока, $\varepsilon=\varepsilon(p, \rho)-$ внутренняя энергия плазмы, $g(x)$ - плотность потока материала стенок в плазму, $f(x)-$ сила, действующая на нее, $q(x)$ - плотность джоулева тепловыделения. В уравнении (2) пренебрегли трением потока о стенку, а в уравнении (3) - теплообменом между ними.

Из системы (1)-(3) следует уравнение для скорости потока:

$$
\frac{1}{2 u^{2}}\left(c_{s}^{2}-u^{2}\right) \frac{d u^{2}}{d x}=F
$$

где $c_{s}-$ скорость звука:

$$
c_{s}=\sqrt{\left(\frac{\partial p}{\partial \rho}\right)_{S}}=\sqrt{\left(\left(p / \rho^{2}-\varepsilon_{\rho}\right) / \varepsilon_{p}\right)} .
$$

В формуле (5) $\varepsilon_{\rho}$ и $\varepsilon_{p}-$ частные производные внутренней энергии $\varepsilon(p, \rho)$ по плотности и давлению соответственно.

Правая часть уравнения (4) находится из выражения

$$
\begin{aligned}
F= & \frac{1}{\rho^{2} \varepsilon_{p}}\left(\frac{q}{u}-f\left(1+\rho \varepsilon_{p}\right)-\rho^{2} c_{s}^{2} \varepsilon_{p} \frac{1}{A} \frac{d A}{d x}\right. \\
& \left.-\frac{g}{u}\left(\varepsilon+\frac{u^{2}}{2}+\rho \varepsilon_{\rho}\right)\right) .
\end{aligned}
$$

Для рассматриваемой модели течения уравнение (4) представляет собой известный закон „обращения воздействия“ в условиях совместного действия „расходного“, „механического“, „теплового“ и геометрического сопла [8]. Для получения трансзвукового течения при $u=c_{s}$ функция $F$ из (6) должна обратиться в нуль, т.е. при переходе скорости потока через скорость звука суммарное внешнее воздействие должно изменить знак. Важную роль в получении трансзвукового течения играет геометрия канала (сопло Лаваля). Сечение канала МПУ является постоянным, поэтому геометрическое сопло „не работает“. Другие факторы, влияющие на течение $(g(x), f(x)$ и $q(x))$, не изменяют своего знака по длине потока, причем они характеризуются примерно одинаковой зависимостью от тока.

Таким образом, даже в отсутствии МТ в канале МПУ весьма сложно получить сверхзвуковое течение плазмы, что и послужило обоснованием для гипотезы [3-5].

Скорость звука $c_{s}$ зависит от температуры $T$, давления и состава плазмы. Характерные значения температуры и давления в канале МПУ можно оценить на основании имеющихся экспериментальных данных, количественный состав плазмы (определяемый в значительной степени испарением стенок канала) неизвестен. В большинстве конструкций МПУ использовались медные электроды и изоляторы из поликарбоната. Соответственно в канал МПУ поступают относительно тяжелые атомы меди и легкие атомы водорода, углерода и кислорода из изоляционной стенки. Средний молекулярный вес плазмы, образующейся при диссоциации продуктов абляции поликарбоната, составляет $\mu_{m} \cong 7$ [5].

В работах $[4,5]$ приведены расчеты по формуле (5) скорости звука в двухкомпонентной медно-углеродной плазме. Принималось, что углерод моделирует испарение изолятора. При характерных значениях параметров плазмы $p \sim 0.1 \mathrm{GPa}$ и $T \sim 30 \mathrm{kK}$ (см. ниже) в чисто „медной“ плазме скорость звука составляет $3.9 \mathrm{~km} / \mathrm{s}$, в „Углеродной“ плазме $-7.4 \mathrm{~km} / \mathrm{s}$, при равной концентрации атомов меди и углерода $-5.4 \mathrm{~km} / \mathrm{s}$. Таким образом, достигнутые скорости метания [1] находятся внутри диапазона вероятных значений скорости звука. Этот факт косвенно подтверждает справедливость гипотезы [3-5].

Скорость звука можно увеличить, если использовать графитовые электроды, однако по своим электромеханическим свойствам обычные графиты не годятся для электродов МПУ. По совокупности электрофизических свойств для электродов МПУ лучше всего подходит бериллий. По расчетам скорость звука в „бериллиевой“ плазме при $p \sim 0.1 \mathrm{GPa}$ и $T \sim 30 \mathrm{kK}$ составляет $9.5 \mathrm{~km} / \mathrm{s}$ [5]. Еще более высокие скорости звука достигаются в литиевой плазме. По данным работы [10] при $p \sim 0.1 \mathrm{GPa}$ и $T \sim 30 \mathrm{kK}$ скорость звука в плазме лития составляет около $10.5 \mathrm{~km} / \mathrm{s}$. Применение лития в качестве электродов исключено, но его можно использовать для нанесения на них покрытий, либо как инициатор разряда.

В плазме для оценки скорости звука от определяющих параметров можно использовать соотношение

$$
c_{s} \propto \sqrt{T / \mu_{m}} .
$$

Из данных работы [10] и соотношения (7) следует, что если использовать инициатор из гидрида лития $\left(\mu_{m}=4\right)$, то при $p \sim 0.1 \mathrm{GPa}$ и $T \sim 30 \mathrm{kK}$ скорость звука достигнет $\sim 14 \mathrm{~km} / \mathrm{s}$. Эта скорость увеличится до $\sim 15 \mathrm{~km} / \mathrm{s}$, если в этом соединении использовать изотоп ${ }^{6} \mathrm{Li}$.

В работе [7] исследовалось движения свободного ПП (СПП) в канале рельсотрона, заполнявшегося гелием $(\mathrm{He})$, воздухом или ксеноном (Хе). Форма импульса тока в МПУ была близка к трапеции - ток возрастал до $450 \mathrm{kA}$ за $\sim 20 \mu \mathrm{s}$, потом в течение $\sim 40 \mu \mathrm{s}$ удерживался на этом уровне. Скорость СПП увеличивалась на стадии роста тока, затем она оставалась постоянной, пока ток не снижался. Для скорости СПП были получены значения: $4.6(\mathrm{Xe}), 9.8$ (воздух) и $17.5 \mathrm{~km} / \mathrm{s}(\mathrm{He})$. Авторы работы [7] пришли к выводу, что их результаты „не согласуются с утверждением работы [4] относительно ограничения скорости метаемого тела скоростью звука в плазме. При температуре $30-40 \mathrm{kK}$. . с скорость звука в медно-углеродной плазме не превосходит $7 \mathrm{~km} / \mathrm{s}$, что гораздо ниже максимальных значений скорости, полученных нами в гелии и в воздухе“.

Однако этот вывод представляется спорным, так как в работе [7] движение границы СПП рассматривалось 
в приближении ударной волны. Если зазор между заглушкой канала и МТ достаточно велик, то в канале рельсотрона действительно может возникнуть ударная волна, но после ее столкновения с МТ характер течения плазмы радикально изменится.

Было бы полезно сопоставить скорость СПП из работы [7] со скоростью звука, однако для этого недостаточно данных. Скорость звука увеличивается с температурой, которая возрастает при увеличении темпа ввода энергии в МПУ, т.е. с ростом производной тока. В опытах [7] на стадии разгона СПП темп роста тока составлял $\sim 25 \mathrm{kA} / \mu \mathrm{s}$, в то время как в типичных режимах рельсотронов с МТ этот темп в 5-10 раз меньше (см., например, работы $[11,12]$ ). Поэтому температура плазмы в условиях опытов [7] и скорость $c_{s}$ могли быть достаточно велики. Если принять, что температура СПП не зависела от среды, то скорость звука по соотношению (7) обратно пропорциональна $\mu_{m}^{1 / 2}$. Если в гелии скорость СПП $(17.5 \mathrm{~km} / \mathrm{s})$ равнялась скорости звука, то в воздухе эта скорость при полной диссоциации его молекул должна была равняться $9.1 \mathrm{~km} / \mathrm{s}$, a в Xe $-3.1 \mathrm{~km} / \mathrm{s}$. Эти значения близки к скорости СПП из работы [7], особенно если учесть, что в плазме ксенона продукты эрозии стенок, поступающие в канал, уменьшая молекулярный вес $\mu_{m}$, увеличивают скорость звука.

\section{2. Экспериментальная установка}

В настоящей работе в основном обсуждаются эксперименты по метанию в МПУ ударников массой $m_{b}$ до $3 \mathrm{~g}$. Наши эксперименты по метанию „тяжелых“ ударников массой $15 \mathrm{~g}$ описаны в работе [13]. В работе [14] представлены результаты метания дюралевых ударников массой 60-90 g в рельсотроне с „твердым“ якорем.

В наших опытах использовался МПУ с длиной канала $l_{c}=0.16-1.0 \mathrm{~m}$ и диаметром $d_{c}=12-16 \mathrm{~mm}$. Масса MT, выполненных из поликарбоната, была $1-3$ g. Электроды изготавливались из латуни ЛС-59, изоляторы из поликарбоната. В казенную часть канала вкручивалась заглушка из капролона длиной $6 \mathrm{~cm}$. После сборки канал обкладывался диэлектрическими пластинами и помещался в силовой бандаж. Погонная индуктивность канала составляла $L^{\prime}=0.3-0.4 \mu \mathrm{H} / \mathrm{m}$.

Источником энергии служил емкостной накопитель (EH) из 8 секций емкостью $9.6 \mathrm{mF}$ каждая, имевших независимую систему запуска. Энергия ЕН при максимальном напряжении зарядки $5 \mathrm{kV} W_{0}=0.8 \mathrm{MJ}$. При экспериментах с каналами длиной 0.7 и $1.0 \mathrm{~m}$ рельсотрон, как правило, подключался к ЕН через формирующую линию с индуктивностью $0.3 \mu \mathrm{H}$ и емкостью $14 \mathrm{mF}$.

В большинстве экспериментов для инициации разряда использовалась литиевая пластинка, соединявшая электроды МПУ. Также проводились опыты с инициатором из графита. Во всех случаях масса инициатора $m_{\text {in }}$ составляла $\sim 10 \%$ от массы МТ.

Схема основных измерений пояснена на рис. 1. Ток $I(t)$ определялся с помощью пояса Роговского,

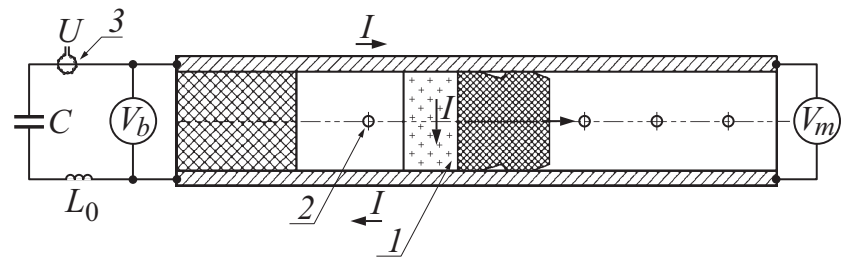

Рис. 1. Схема основных измерений: 1 - плазменный поршень, 2 - магнитные зонды, 3 - пояс Роговского.

напряжение на входе в канал $V_{b}(t)$ и на плазме $V_{m}(t)-$ с помощью трансформаторов тока. Погрешность измерения напряжения и тока составляли 5 и $10 \%$ соответственно. Движение ПП внутри канала контролировалось при помощи 6-8 магнитных зондов (М3), которые были вмонтированы в изоляционную стенку на расстоянии $3 \mathrm{~mm}$ от рабочей поверхности канала.

Во всех экспериментах скорость МТ на баллистической трассе рассчитывалась из времени пролета МТ от выходного сечения канала до мишени. В качестве мишеней использовались дюралевые диски. Момент вылета МТ из канала фиксировался по резкому росту напряжения на плазме $V_{m}$, момент соударения с мишенью контактным датчиком.

В контрольных опытах скорость МТ на баллистической трассе измерялась с помощью „световых“ ножей и скоростной кино- и видеосъемки. Отличие значений скорости МТ, измеренной тремя разными методами, было менее $3 \%$.

\section{3. Результаты экспериментов}

Диапазон скоростей метания um в наших экспериментах составил от $2.1-5.4 \mathrm{~km} / \mathrm{s}$. Максимальная скорость была получена в канале с размерами $l_{c}=0.7 \mathrm{~m}$ и $d_{c}=13 \mathrm{~mm}$; масса МТ равнялась $1.4 \mathrm{~g}$. Основная часть опытов проводилась с каналами длиной 0.16 и $0.47 \mathrm{~m}$, максимальная скорость МТ при этом была 3.5 и $4.7 \mathrm{~km} / \mathrm{s}$. При более „жестких“ режимах разгона происходило разрушение МТ, самые быстрые фрагменты МТ имели скорость на $\sim 5 \%$ больше, чем были достигнуты при неразрушаемом ускорении.

Далее подробно разбирается эксперимент, в котором MT массой $2.6 \mathrm{~g}$ было ускорено до скорости $5.2 \mathrm{~km} / \mathrm{s}$, использовался литиевый инициатор $m_{\text {in }}=0.2 \mathrm{~g}$. Диаметр канала - $16 \mathrm{~mm}$, его длина - $1 \mathrm{~m}$. Использовалось 8 секций ЕН при зарядном напряжении $3.9 \mathrm{kV}$, $W_{0}=580 \mathrm{~kJ}$. На рис. 2 приведены осциллограммы тока и напряжений для этого эксперимента. Из данных на рис. 2 рассчитывались значения энергии, подведенной к каналу $Q_{b}$ и к плазме $Q_{m}$. К моменту вылета МТ из канала $(\sim 390 \mu \mathrm{s})$ значения этих энергий составили $Q_{b}=210 \mathrm{~kJ}$ и $Q_{m}=74 \mathrm{~kJ}$. Кинетическая энергия МТ на вылете из канала равнялась $W_{k}=35 \mathrm{~kJ}$, т.е. $6 \%$ энергии EH перешло в кинетическую энергию МТ. Эффективность преобразования энергии, подведенной к каналу $Q_{b}$, в кинетическую энергию МТ $-17 \%$. 


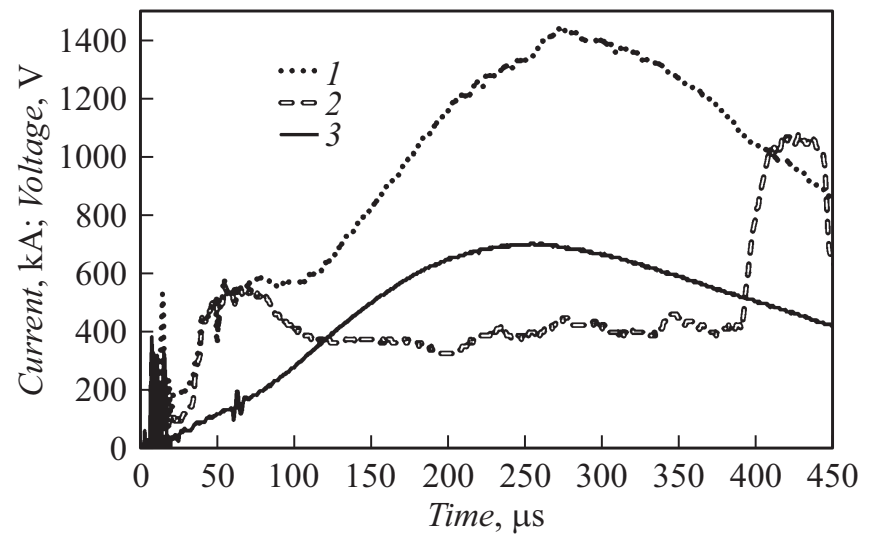

Рис. 2. Осциллограммы входного напряжения $V_{b}(1)$, напряжения на выходе $V_{m}(2)$ и тока (3).

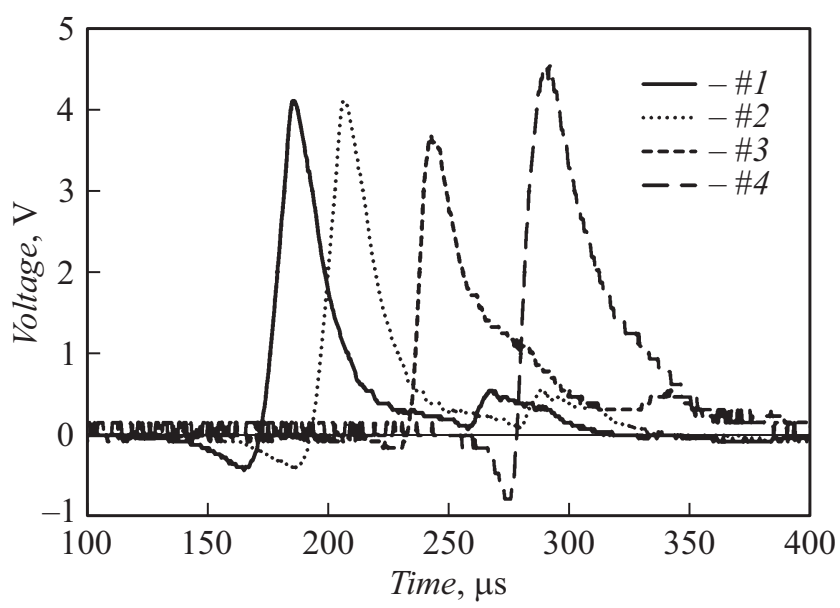

Рис. 3. Сигналы с магнитных зондов.

Из анализа данных на рис. 2 определяется не только момент вылета МТ из канала, но и время его старта, правда, менее точно (см. ниже). В обсуждаемом эксперименте МТ пришло в движение в момент времени $t_{0} \cong 80 \mu \mathrm{s}$.

На рис. 3 представлены осциллограммы сигналов с первых четырех M3, установленных в канале. На рис. 4 по показаниям М3 построена $x(t)$ - диаграмма движения ПП, на основании которой можно судить об $x(t)-$ диаграмме MT.

На рис. 5 показана скорость ПП в канале, рассчитанная из данных рис. 4. На этом рисунке также приведена скорость МТ в момент вылета из канала. Как видно из рис. 5, в канале рельсотрона происходит торможение ПП. Подобное поведение ПП являлось типичным для наших экспериментов. На рис. 6 приведены профили скорости ПП внутри канала, полученные в трех других экспериментах. На этом рисунке также показаны значения скорости МТ при вылете из канала - они составили $4.5\left(m_{b}=1.5 \mathrm{~g}, l_{c}=0.47 \mathrm{~m}\right), 4.9\left(m_{b}=1.9 \mathrm{~g}\right.$, $\left.l_{c}=1.0 \mathrm{~m}\right)$ и $5.4 \mathrm{~km} / \mathrm{s}\left(m_{b}=1.4 \mathrm{~g}, l_{c}=0.7 \mathrm{~m}\right)$; в скобках приведены значения массы ударника и длины канала.
В экспериментах с „холостыми“ выстрелами графитовым инициатором (без МТ) скорость ПП по длине канала изменялась монотонно. ПП разгонялся до предельной скорости на начальном участке длиной около $0.2 \mathrm{~m}$, после чего его скорость слабо изменялась. В этих опытах использовался канал длиной $0.7 \mathrm{~m}$, масса инициатора была $0.1-0.3 \mathrm{~g}$, скорость ПП достигала $\sim 10 \mathrm{~km} / \mathrm{s}$.

Торможение ПП было зафиксировано в наших экспериментах по метанию „тяжелых“ ударников при помощи магнитного зонда в виде витка шириной $7 \mathrm{~mm}$, который был установлен вдоль всей изоляционной стенки [13]. На осциллограмме напряжения на зонде при растущем токе в МПУ было зарегистрировано плато длительностью около $20 \mu \mathrm{s}$, что свидетельствует о торможении ПП. Скорость МТ в этот момент, по оценкам, была около $2 \mathrm{~km} / \mathrm{s}$. Скорость МТ метания в этих экспериментах равнялась $3 \mathrm{~km} / \mathrm{s}$, использовался канал длиной $1.0 \mathrm{~m}$ и диаметром $24 \mathrm{~mm}$.

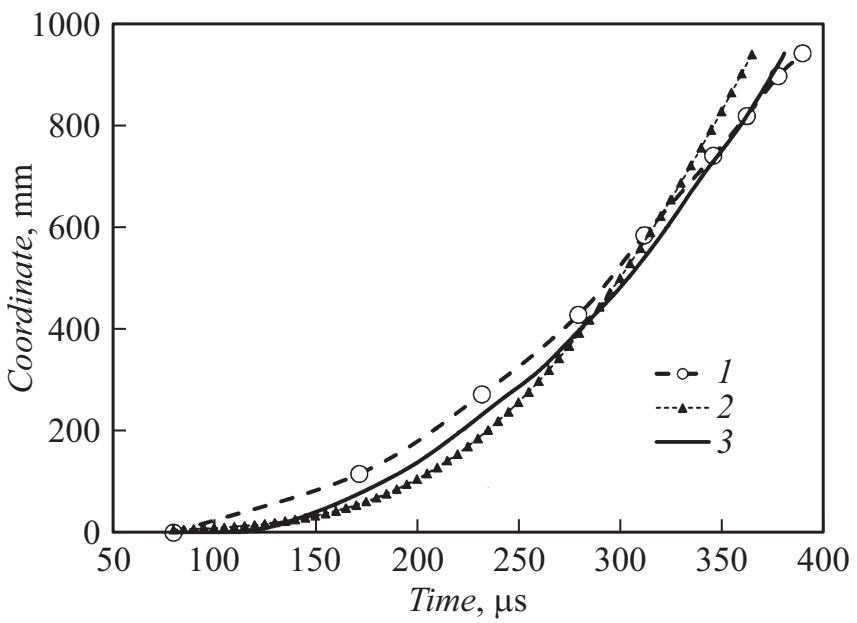

Рис. 4. $X(t)$-диаграмма: $1-$ ПП из данных рис. $3 ; 2-$ МТ из формулы (13); 3 - ПП из формулы (15).

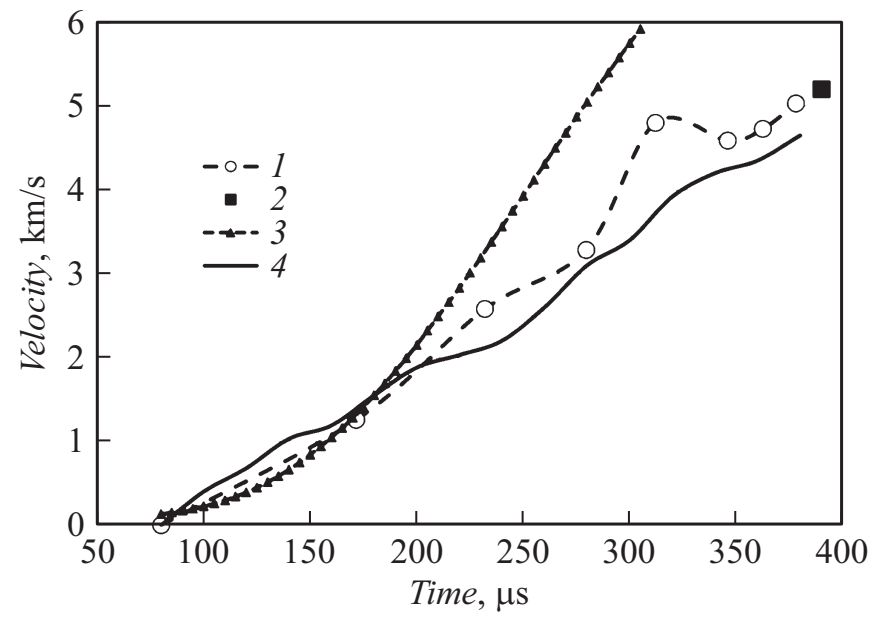

Рис. 5. Скорость ПП и МТ внутри канала: ПП - из данных рис. 3 (1) и формулы (15) (2), МТ - из формулы (14). 


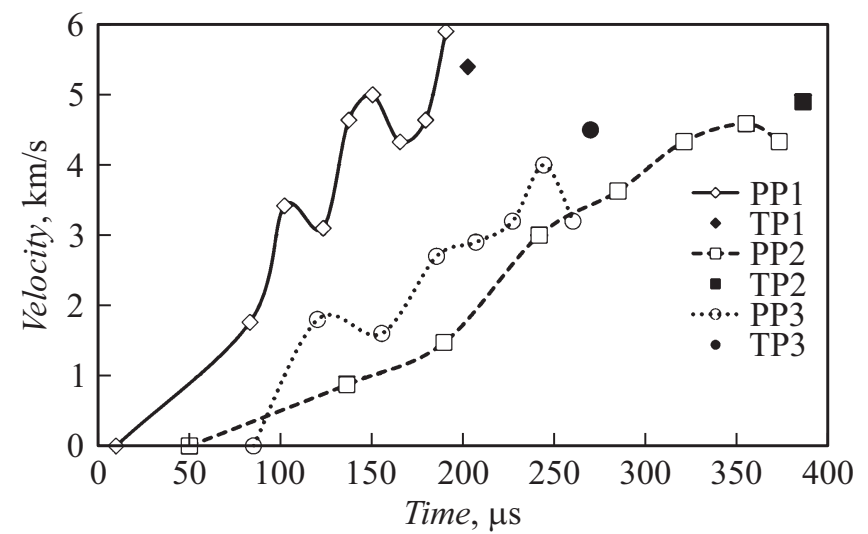

Рис. 6. Скорость ПП внутри канала (прозрачные маркеры) и МТ на вылете из канала (сплошные маркеры).

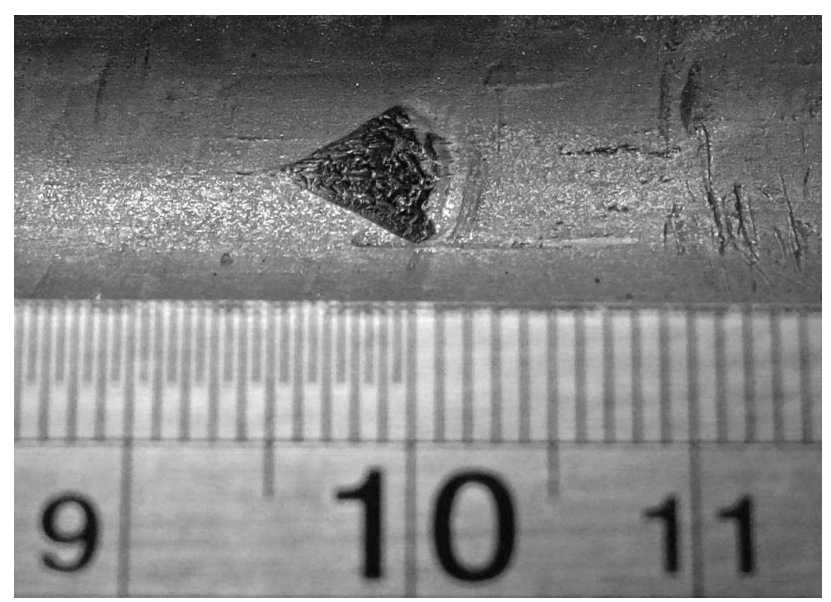

Рис. 7. Повреждение на поверхности изолятора.

Отметим, что торможение ПП и его отставание от МТ в канале МПУ регистрировались в самых разных экспериментальных условиях $[1,11,15]$.

Во время „выстрела“ стенки канала испытывали экстремальные тепловые и динамические нагрузки, вследствие чего, как правило, его повторное использование было исключено. На начальном участке электродов длиной $\sim 100$ mm наблюдалась зона расплава, на остальной их части следы плавления не были столь выражены. Изоляторы не имели явных следов термического воздействия, однако на их поверхности наблюдались специфические „каплеобразные“ повреждения („задиры“) глубиной до $0.5 \mathrm{~mm}$. Эти повреждения фиксировались в основном в центральной части канала, где скорость МТ по оценкам изменялась от 2 до $3 \mathrm{~km} / \mathrm{s}$. Вблизи выходного сечения канала интенсивность повреждений изолятора была существенно меньше, на начальном участке они отсутствовали. На рис. 7 приведен участок поверхности изолятора, где скорость МТ была $\sim 3 \mathrm{~km} / \mathrm{s}$.

Аналогичные повреждения центрального участка канала МПУ приведены в работе [13]. Время прохождения МТ этого участка канала соответствовало моменту торможения ПП, описанному выше.
Существование специфических повреждений на поверхности канала рельсотрона неоднократно отмечалось в литературе. В работе [16] описаны повреждения на электродах в центральной части канала, наблюдавшихся в экспериментах [11]. Для этих повреждений в работе [16] использовался термин „gouging“, т.е. след, оставляемый полукруглой стамеской. Судя по описаниям, такие же повреждения на изоляторах или электродах были зарегистрированы в экспериментах [17-19]. В опытах [17] продольный размер этих следов достигал 10-15 mm, в экспериментах [18] он был вдвое меньше. По данным работ $[16,18,19]$ повреждения появлялись в центральной части канала, где скорость ударников по оценкам была от 1.5 до $4 \mathrm{~km} / \mathrm{s}$. На более скоростном участке канала они либо отсутствовали, либо их количество заметно уменьшалось. В работе [16] обсуждалась гипотеза, связывающая эти повреждения со столкновениями МТ с небольшими частицами, находящимися на поверхности канала.

Отметим, что в работе [20] приведена фотография „следа“, который остался на медном электроде при скольжении по нему дюралевого ударника со скоростью свыше $1.5 \mathrm{~km} / \mathrm{s}$ (рельсотрон с „твердым“ якорем). Этот „след“ подобен повреждению на рис. 7.

\section{4. Анализ экспериментальных данных}

\section{1. Давление и температура в ПП}

Характерную величину давления плазмы в канале МПУ можно оценить из среднего ускорения МТ

$$
p \approx 2 m_{b} u_{m}^{2} /\left(\pi l_{c} d_{c}^{2}\right) .
$$

Для условий обсуждаемого эксперимента это давление составляло $p \sim 0.2 \mathrm{GPa}$, максимальное давление было вдвое больше. Приведем для сравнения оценки из (8) характерного давления для экспериментов, известных из литературы. При характерной скорости $u_{m} \sim 5 \mathrm{~km} / \mathrm{s}$ давление плазмы для экспериментов [11] составило 60-80 MРа, для экспериментов [21] - 250-550 МРа.

Температуру ПП можно оценить по максимуму. Энергия, выделяющаяся в ПП, расходуется на тепловое излучение, ионизацию продуктов эрозии стенок, расширение плазмы и т.д. Поэтому в центральной части ПП справедливо соотношение

$$
j V_{m} \approx d_{c} j^{2} / \sigma(T)>2 \varepsilon \sigma_{0} T^{4},
$$

где $j-$ плотность тока в ПП, $\sigma_{0}=$ $=5.67 \mathrm{~W} /\left(\mathrm{cm}^{2} \cdot \mathrm{kK}^{4}\right)$ - постоянная Стефана-Больцмана, $\varepsilon$ и $\sigma(T)$ - степень „черноты“ плазмы и ее электропроводность. Для МПУ можно принять, что электропроводность плазмы описывается формулой Спитцера [22] $\left(\sigma(T)=a T^{3 / 2}\right)$, а степень ее „черноты близка к единице, тогда из (9) следует оценка для максимальной температуры плазмы:

$$
\left(d_{c} k^{2} /\left(2 a \sigma_{0}\right)\right)^{2 / 11}>T_{\max }
$$


При $p \sim 0.1 \mathrm{GPa}$ коэффициент пропорциональности в формуле Спитцера $a \cong 1.21 /\left(\Omega \cdot \mathrm{cm} \cdot \mathrm{kK}^{3 / 2}\right)$. В наших экспериментах $j \sim 50 \mathrm{kA} / \mathrm{cm}^{2}$, тогда из (10) следует, что $T_{\max } \sim 35 \mathrm{kK}$. Температура $T_{\max }$ слабо зависит от плотности тока и практически не зависит от диаметра канала, поэтому она, по-видимому, не может превысить $35-40 \mathrm{kK}$. По данным работы [1] температура ПП в начальный момент работы МПУ изменялась в пределах $25-30 \mathrm{kK}$

При давлении $p \sim 0.2 \mathrm{GPa}$ и температуре $T \sim 30 \mathrm{kK}$ концентрация частиц в полностью ионизованной плазме составляет $\sim 2 \cdot 10^{20} \mathrm{~cm}^{-3}$.

\section{2. Динамика МТ и ПП в канале}

В пренебрежении потерями импульса и массы ПП ускорение МТ и его скорость рассчитываются из уравнений [1]:

$$
\begin{gathered}
\ddot{x}=L^{\prime} I^{2} /\left(2 m_{b}\right), \\
u(t)=u_{0}+L^{\prime} \int I^{2}\left(t^{\prime}\right) d t^{\prime} /\left(2 m_{b}\right),
\end{gathered}
$$

где $u_{0}-$ скорость МТ на входе в канал.

Скорость МТ, рассчитанная из соотношения (12), является максимально возможной. Эффективность работы МПУ можно характеризовать коэффициентом передачи импульса (КПИ) $\eta_{m}[12]$, который представляет собой отношение реального увеличения скорости МТ в канале к расчетному значению, определяемому из формулы (12).

По рассчитанной скорости находится координата МТ

$$
x_{b}(t)=u_{0} t+\int u\left(t^{\prime}\right) d t^{\prime}
$$

Скорость МТ на входе в канал может быть достаточно велика. В экспериментах [11] предварительный разгон МТ на входе в канал до скорости $\sim 1 \mathrm{~km} / \mathrm{s}$ осуществлялся с помощью сжатого гелия. В наших экспериментах МТ получало начальное ускорение под действием давления паров лития. К моменту старта МТ $\left(t_{0} \cong 80 \mu \mathrm{s}\right)$ в инициаторе выделилась энергия $Q_{m} \cong 3 \mathrm{~kJ}$, а ток достиг $\sim 100 \mathrm{kA}$. Для испарения лития требуется $\sim 20 \mathrm{~kJ} / \mathrm{g}[23]$, поэтому испарилась и ионизовалась только часть инициатора. Инициатор разрушился вследствие механических напряжений, которые к моменту старта МТ превысили $10 \mathrm{MPa}$.

На рис. 4 приведены рассчитанные из интеграла 13) значения координаты МТ для обсуждаемого эксперимента $\left(u_{0}=0\right)$. Как видно, в начальный момент МТ отставало от ПП. Этот парадокс объясняется тем, что при этом расчете скорости не учитывалось дополнительное ускорение, которое МТ получило под действием давления паров лития. Формально этот эффект можно учесть заданием „начальной“ скорости МТ. Из данных рис. 4 следует, что в результате этого механизма МТ приобрело скорость $u_{0} \cong 0.6 \mathrm{~km} / \mathrm{s}$, кинетическая энергия при этом составила $W_{k} \cong 0.5 \mathrm{~kJ}$. Максимальная „начальная" скорость МТ достигала $0.8 \mathrm{~km} / \mathrm{s}$. В наших опытах с короткоствольным рельсотроном $\left(l_{c}=0.16 \mathrm{~m}\right)$ при скорости метания $u_{m}$ до $3 \mathrm{~km} / \mathrm{s}$ она, как правило, на $\sim 10 \%$ превышала значение, определяемое интегралом (12). Соответственно „начальная“ скорость МТ в этих экспериментах была не менее $0.3 \mathrm{~km} / \mathrm{s}$.

На рис. 5 приведена скорость МТ внутри канала, рассчитанная из интеграла (12). К моменту вылета скорость МТ должна была достигнуть $8.2 \mathrm{~km} / \mathrm{s}$, так что КПИ в этом эксперименте равнялся 63\%. По литературным данным при скорости метания $5.2 \mathrm{~km} / \mathrm{s}$ КПИ находится в диапазоне от 40 до 80\% [12]. Из данных рис. 2 и 5 следует, что на стадии спада тока в ускорителе МТ набрало 30-40\% от результирующей скорости.

Представление о динамике ПП можно также получить из анализа осциллограмм тока и напряжений. Если рассматривать ПП как токовую перемычку, то второй закон Кирхгофа для МПУ имеет вид

$$
V_{b}-V_{m}-I R(t)=L^{\prime} \frac{d}{d t} I\left(x_{0}+x_{p}\right),
$$

где $x_{0}$ - длина начального участка канала (заглушки), $R(t)$ - сопротивление электродов.

Из уравнения (14) оценивалось время старта МТ. Так как падение напряжения на электродах в начальный момент времени мало, из уравнения (14) следует, что пока МТ покоится разность напряжений $V_{b}$ и $V_{m}$ равна ЭДС индукции на начальном участке канала. Когда МТ приходит в движение, это условие перестает выполняться.

По оценкам в первом приближении падением напряжения на электродах можно пренебречь, тогда из уравнения (14) следует соотношение для координаты ПП

$$
x_{p}(t)=\frac{1}{L^{\prime} I(t)} \int\left(V_{b}\left(t^{\prime}\right)-V_{m}\left(t^{\prime}\right)\right) d t^{\prime}-x_{0} .
$$

На рис. 4 приведена $x(t)$-диаграмма ПП, рассчитанная по формуле (15). Как видно, эта $x(t)$-диаграмма согласуется с данными МЗ. Из дифференцирования зависимости (15) находится скорость ПП, которая в „сглаженном“ виде показана на рис. 5. При скорости до $3 \mathrm{~km} / \mathrm{s}$ относительные пульсации ее значений были менее $10 \%$, при скорости ПП свыше $4 \mathrm{~km} / \mathrm{s}$ эти пульсации достигали $\sim 30 \%$. С учетом этого данные о скорости ПП, полученные из соотношения (15), неплохо согласуются с данными МЗ. В целом уравнение (15), следующее из закона Кирхгофа при вполне реалистичных предположениях, может использоваться для контроля динамики ПП в МПУ.

Уравнение (14) может быть проинтегрировано в квадратурах, если принять, что сопротивление рельсов пропорционально координате ПП: $R(t)=R^{\prime}\left(x_{p}(t)+x_{0}\right)$, где $R^{\prime}$ - погонное сопротивление электродов. Тогда решение уравнения (14) имеет вид

$$
x_{p}(t)=\frac{\exp (-t)}{R^{\prime} I(t)} \int \exp \left(t^{\prime}\right)\left(V_{b}\left(t^{\prime}\right)-V_{m}\left(t^{\prime}\right)\right) d t^{\prime}-x_{0} .
$$


В формуле (16) за единицу времени принято отношение $R^{\prime} / L^{\prime}$. Как показывает анализ, использование более сложного соотношения (16) не имеет явного преимущества перед формулой (15), тем более что вследствие скин-эффекта по оценкам за время „выстрела“ погонное сопротивление $R^{\prime}$ изменяется примерно втрое.

Из рис. 5 следует, что в обсуждаемом эксперименте торможение ПП происходило при скоростях около 2.5 и $4.5 \mathrm{~km} / \mathrm{s}$. Аналогичное поведение ПП наблюдалось и в других экспериментах, представленных на рис. 6: торможение ПП происходило в диапазоне скоростей 1-3 и $4-5 \mathrm{~km} / \mathrm{s}$. Скорость ПП по данным МЗ определяется со значительной погрешностью, однако торможение ПП при разгоне МТ прямо подтверждается результатами работы [13], а также наличием повреждений на изоляторе и динамикой СПП при „холостых“ выстрелах.

Применение массивного инициатора из лития или графита позволило создать на начальном участке канала плотную плазму с высокой скоростью звука, под действием давления которой МТ получало скорость $\sim 0.5 \mathrm{~km} / \mathrm{s}$. В известных экспериментах $[11,12]$ получение „легкой“ плазмы на входе в канал достигалось использованием сжатого гелия, обеспечивавшего начальный разгон МТ до скорости $0.6-1.2 \mathrm{~km} / \mathrm{s}$. Применение массивного легкоплазменного инициатора позволило решить эту задачу более простым способом.

В большинстве известных конструкций МПУ для инициации разряда использовалась металлическая фольга, масса которой составляла $\sim 1 \%$ от массы МТ. Однако ускорение МТ в МПУ происходит под действием давления ПП, при этом концентрация частиц в нем составляет $\sim 1 \cdot 10^{20} \mathrm{~cm}^{-3}$. При использовании ,Легкого“ инициатора невозможно обеспечить такую концентрацию частиц в протяженном ПП. Поэтому испарение и абляция стенок канала могут являться фактически необходимым условием работы МПУ. Массивный инициатор позволяет получить ПП с требуемой концентрацией частиц, при этом высокое давление образовавшейся плазмы частично блокирует испарение стенок канала.

\section{5. Резонансная деформация канала рельсотрона}

Представленные результаты позволяют сделать вывод о влиянии деформации канала на эффективность работы МПУ. Впервые эта гипотеза обсуждалась в докладе [24]; результаты настоящей работы существенно дополняют аргументы, приведенные в [24].

Известно, что динамическая деформация, создаваемая движущейся нагрузкой, может значительно превосходить статическую, при этом возможно возникновение резонансных явлений [25]. Расчет деформации канала МПУ с учетом изоляционной стенки и силового бандажа представляет собой чрезвычайно сложную задачу, поэтому ограничимся рассмотрением простой модели - деформации цилиндра под действием движущегося фронта высокого давления. Из теории оболочек известно, что
Значения критических скоростей для ряда металлов

\begin{tabular}{c|c|c|c|c}
\hline \multirow{2}{*}{ Металл } & \multicolumn{2}{|c|}{$V_{c r 1}, \mathrm{~km} / \mathrm{s}$} & \multicolumn{2}{c}{$V_{c r 2}, \mathrm{~km} / \mathrm{s}$} \\
\cline { 1 - 5 } & $h / a=0.5$ & $h / a=1$ & $h / a=0.5$ & $h / a=1$ \\
\hline Бериллий & 6.84 & 9.68 & 12.7 & 12.7 \\
Вольфрам & 2.42 & 3.40 & 4.66 & 4.72 \\
Медь & 2.02 & 2.83 & 3.99 & 4.07 \\
Молибден & 3.04 & 4.27 & 5.87 & 5.95 \\
Сталь & 2.76 & 3.88 & 5.29 & 5.35
\end{tabular}

в этом случае существуют две критические скорости фронта, при которых происходит резкое увеличение амплитуды колебаний стенок канала [26] - развивается резонансная деформация канала (РДК). Первая критическая скорость связана с колебаниями, изгибающими канал, вторая - с нарастанием продольных колебаний. В работе [26] из анализа деформация цилиндрической оболочки под действием нагрузки, движущейся с постоянной скоростью, получены следуюшие соотношения для критических скоростей:

$$
\begin{gathered}
V_{c r 1}^{2}=\frac{h E}{\rho a \sqrt{3\left(1-\mu^{2}\right)}}\left(1-\frac{h \mu^{2}}{a \sqrt{12\left(1-\mu^{2}\right)}}\right), \\
V_{c r 2}^{2}=\frac{E}{\rho\left(1-\mu^{2}\right)}\left(1+\frac{h^{2} \mu^{2}}{3 a^{2}\left(1-\mu^{2}\right)}\right),
\end{gathered}
$$

где $a-$ средний радиус цилиндра, $h-$ толщина стенки, $\rho$ - плотность материала оболочки, $E-$ модуль Юнга, $\mu$ - модуль Пуассона. Формулы (17) и (18) были выведены при условии, что $h / a \ll 1$; из численных расчетов методом конечных элементов [27] следует, что при $h / a \cong 1$ эти формулы завышают значения критических скоростей на $\sim 10 \%$.

Как видно из формул (17) и (18), критические скорости возрастают примерно пропорционально характерной скорости звука в стенках канала $c_{w} \sim(E / \rho)^{0.5}$.

В таблице приведены значения $V_{c r 1}$ и $V_{c r 2}$, рассчитанные по формулам (17) и (18) при двух значениях относительной толщины стенки $h / a$ для металлов, использовавшихся для электродов МПУ. В таблицу добавлены данные для бериллия, имеющего максимальные критические скорости. Свойства металлов $(\rho, E, \mu)$ взяты из справочника [23]. С учетом сложной конструкции МПУ значения критических скоростей, приведенные в таблице, можно рассматривать как ориентировочные.

Влияние РДК на эффективность работы МПУ проявляется по следующей схеме: заметно увеличивается деформация канала, и на его поверхности могут возникать динамические неоднородности, при столкновении МТ с которыми происходит взрывной выброс материала стенок и МТ в плазму. Как следствие, ПП резко тормозится, при этом уменьшается температура и электропроводность плазмы. Торможение ПП вызывает скачок входного напряжения, который может спровоцировать пробои на входе в рельсотрон и возникновение паразитных токовых структур в следе ПП. 
Торможение ПП сменяется его разгоном под действием пондеромоторных сил, величину ускорения можно оценить из данных рис. 5 и 6. Максимальное ускорение ПП $\left(\sim 10^{8} \mathrm{~m} / \mathrm{s}^{2}\right)$ достигалось в эксперименте с максимальной скоростью метания $(5.4 \mathrm{~km} / \mathrm{s})$. Масса ПП, оцениваемая из уравнения (11), равнялась $\sim 0.3 \mathrm{~g}$. МТ после торможения ПП продолжало двигаться по инерции, пока ПП не догонял его. Импульс разогнавшегося ПП частично передается МТ, поэтому негативное влияние РДК на работу МПУ может сводиться в основном к увеличению массы ПП и снижению его температуры.

В рамках рассматриваемой модели РДК следует ожидать снижения эффективности разгона МТ, когда его скорость находится в диапазоне между критическими скоростями. На качественном уровне этот вывод согласуется с имеющимися литературными данными и нашими результатами. При малых, до $\sim 3 \mathrm{~km} / \mathrm{s}$, скоростях МТ потери импульса в рельсотроне малы $\left(\eta_{m} \cong 90 \%\right)$, а основное снижение эффективности работы МПУ фиксировалось при скоростях метания свыше $3-4 \mathrm{~km} / \mathrm{s}$ [1,11,12,15]. Отметим, что если следовать этой гипотезе, то при скоростях метания свыше второй критической можно ожидать снижения потерь импульса. Результаты работы [11] подтверждают это предположение: максимальное значение КПИ (80\%) было получено при скорости метания $5.2 \mathrm{~km} / \mathrm{s}$, а при скоростях метания 4.2 и $5.9 \mathrm{~km} / \mathrm{s}$ значения КПИ составили 66 и 60\% соответственно. Применительно к этим результатам гипотеза об ограничении скорости метания значением скорости звука в ПП [3-5], по-видимому, выполняется, так как плазмообразующая среда в экспериментах [11] представляла собой гелий, разбавленный продуктами эрозии стенок канала.

Возникновение РДК при умеренных скоростях МТ в диапазоне от $\sim 2$ до $\sim 4 \mathrm{~km} / \mathrm{s}$ подтверждается обсуждавшимся выше торможением ПП и появлением „задиров“ на поверхности канала. В литературе известны и другие особенности характеристик МПУ, которые можно интерпретировать, как проявление РДК, а именно

- увеличение деформации канала;

- большой разброс результатов значений КПИ при скоростях метания вблизи резонансной скорости;

- влияние механических свойств материала электродов на скорость метания;

- разрушение МТ внутри канала МПУ.

В экспериментах [18] измерялась зависимость от времени смещения внешней стенки медного электрода МПУ. По данным работы [18], через $0.22 \mathrm{~ms}$ после начала разгона происходило резкое увеличение смещения стенки, достигавшее $0.25 \mathrm{~mm}$, при этом зависимость смещения от времени имело вид нарастающих колебаний. Максимальное смещение $(0.34 \mathrm{~mm})$ фиксировалось через $0.35 \mathrm{~ms}$ после начала движения MT.

В работе [12] представлена серия экспериментов, в которых использовались электроды из меди и молибдена, при этом были получены максимальные скорости метания соответственно 4.3 и $5.5 \mathrm{~km} / \mathrm{s}$. В канале с медными электродами при увеличении скорости МТ возрастал разброс значений КПИ. При скорости $3.5 \mathrm{~km} / \mathrm{s}$ значения КПИ были заключены в диапазоне от 60 до $70 \%$, а вблизи максимальной скорости $(4.3 \mathrm{~km} / \mathrm{s})$ КПИ изменялся от 30 до $60 \%$. Анализ, приведенный в [24], показал, что в экспериментах [12] торможение ПП и его отставание от МТ наблюдались на медных и молибденовых электродах при скоростях соответственно $\cong 4.3$ и $\cong 5.5 \mathrm{~km} / \mathrm{s}$. Значения этих скоростей близки к ориентировочным значениям второй критической скорости, приведенных в таблице.

Во время разгона МТ испытывает экстремальные инерционные нагрузки, которые нередко превышают предел прочности материала, поэтому возможен распад МТ после вылета из канала. Однако разрушение МТ внутри канала, наблюдавшееся, например, в экспериментах $[12,21,28]$, может быть связано с внешним воздействием на МТ.

В нашей работе [29] приведены результаты исследования электротермического ускорителя (ЭТУ), в котором дюралевый ударник массой $70 \mathrm{mg}$ разгонялся в стальном цилиндрическом канале с внутренним диаметром $3 \mathrm{~mm}$ и наружным $18 \mathrm{~mm}$. Достигнутая скорость метания составила $4.2 \mathrm{~km} / \mathrm{s}$. Для ЭТУ в отличие от МПУ значения критических скоростей, рассчитанные из соотношений (17) и (18), являются достаточно точными. Для конструкции канала ЭТУ из работы [29] первая критическая скорость составляла $4.6 \mathrm{~km} / \mathrm{s}(h / a=1.4)$. Как отмечалось выше, для толстостенного канала расчет по приближенной формуле (17) завышает значение критической скорости на $\sim 10 \%$. С учетом этого максимальная скорость метания в экспериментах [29] практически совпала со значением первой критической скорости.

В целом приведенные выше факты свидетельствуют в пользу гипотезы о существенном влиянии РДК на характеристики МПУ. Уместно отметить, что с недавних пор РДК под действием движущегося фронта высокого давления рассматривается как один из наиболее вероятных механизмов потери электрического контакта между электродами и МТ в рельсовых ускорителях с „твердым“ якорем [27]. Экспериментальные и теоретические исследования РДК в пороховых пушках представлены в работе [30].

\section{Заключение}

МПУ макротел, в которых скорость метания в настоящее время составляет менее $7 \mathrm{~km} / \mathrm{s}$, по-видимому, не реализовали полностью своих возможностей. Если исходить из гипотезы об ограничении скорости метания скоростью звука в плазме ускорителя [3-5], то можно ожидать, в принципе, получения скорости на уровне $10-12 \mathrm{~km} / \mathrm{s}$. В этом случае по своим характеристикам МПУ заметно превзойдут многоступенчатые легкогазовые пушки.

Для достижения скорости метания на уровне второй космической необходимо в канале рельсотрона иметь 
„горячую“ плазму с низким молекулярным весом. На начальном этапе разгона „легкую“ плазму можно создать, используя графитовый или литиевый инициатор. Высокую температуру плазмы можно обеспечить только при „жестких“ режимах ускорения, характеризуемых высоким темпом ввода энергии в МПУ. Однако в этих условиях обостряется проблема обеспечения динамической прочности канала ускорителя вследствие резонансной деформации под действием движущегося фронта высокого давления. Преодоление негативного влияния этого эффекта, вероятно, потребует принципиально новых конструкторских и технологических решений.

\section{Благодарности}

Авторы признательны академику В.Е. Фортову за интерес к работе и поддержку.

\section{Финансирование работы}

Работа финансировалась в рамках Программы фундаментальных научных исследований государственных академий наук на 2013-2020 годы, номер темы государственной регистрации 0044-2014-0003.

\section{Конфликт интересов}

Авторы заявляют, что у них нет конфликта интересов.

\section{Список литературы}

[1] Швецов Г.А., Анисимов А.Г., Башкатов Ю.Л., Станкевич C.B. Рельсовые электромагнитные ускорители твердых тел. Достижения. Проблемы. Перспективы. Тр. Международного семинара „Гидродинамика высоких плотностей энергии“ / Под ред. Г.А. Швецова. Новосибирск: Издво Института гидродинамики СО РАН, 2004. С. 282-304.

[2] Осташев В.Е., Лебедев Е.Ф., Фортов В.Е. // ТВТ. 1993. T. 31. № 2. C. 313-320.

[3] Лузганов С.Н., Полищук В.П., Фортов В.Е., Шурупов А.B. // Физика экстремальных состояний вещества. Черноголовка. Изд-во ИПХФ РАН, 2002. С. 134.

[4] Лузганов С.Н., Полищук В.П., Шурупов А.В. // Известия АН. Серия Энергетика. 2005. № 1. С. 32-43.

[5] Kozlov A.V., Luzganov S.N., Polistchook V.P., Shurupov A.V. // XII $^{\text {th }}$ International Symposium on Electromagnetic Launch Technology, USA, Snowbird, Utah, 25-28 May, 2004. Selected papers. P. 65-69. DOI: 10.1109/ELT.2004.1398048.

[6] Рутберг Ф.Г., Савватеев А.Ф. Гиперскоростные электроразрядные ускорители макротел. Труды Международного семинара „Гидродинамика высоких плотностей энергии“. / Под ред. Г.А. Швецова. Новосибирск: Институт Гидродинамики СО РАН, 2004. С. 267-281.

[7] Жуков Б.Г., Резников Б.И., Куракин Р.О., Розов С.И. // ЖТФ. 2007. Т. 77. Вып. 7. С. 43-49.

[8] Абрамович Г.Н. Прикладная газовая динамика. М.: Наука, 1969. $824 \mathrm{c}$

[9] Повх И.Л. Техническая гидромеханика. Л.: Машиностроение, 1976. 502 с.
[10] Потапов А.В., Бабкин Г.В. // ТВТ. 1967. Т. 5. № 2. С. 246253.

[11] Hawke R.S., Nellis W.J., Newman G.H, Rego J., Susoeff A.R. // IEEE Trans. on Magnetics. 1986. Vol. 22. N 6. P. 1510-1515. DOI: 10.1109/TMAG.1986.1064716

[12] Jamison K.A., Littrell D.M. // IEEE Trans. on Magnetics. 1995. Vol. 31. N 1. P. $168-173$. DOI: $10.1109 / 20.364708$

[13] Kozlov A.V., Kotov A.V., Polistchook V.P., Shurupov A.V., Shurupov M.A. // J. Physics: Conf. Series. 2017. Vol. 927. P. 012027. DOI: 10.1088/1742-6596/927/1/012027

[14] Козлов А.В., Котов А.В., Петров А.В., Полищук В.П., Шурупов А.В. // Труды Х Всероссийской конференции по физической электронике. Махачкала-2018. Изд-во ДГУ, C. $148-152$.

[15] Scanlon J.J., Battech J.H., Thornhill L.D., Jamison K.A., Littrell D.M. // IEEE Trans. Magnetics. 1993. Vol. 29. N 1. P. 859-864. DOI: $10.1109 / 20.195689$

[16] Barker L.M., Trucano T.G., Susoeff A.R. // IEEE Trans. Magnetics. 1989. Vol. 25. N 1. P. 83-87. DOI: $10.1109 / 20.22510$

[17] Дробышевский Э.М., Жуков Б.Г., Розов С.И., Соколов В.М., Куракин Р.О., Савельев М.А. Материалы I Всесоюзного семинара по динамике сильноточного дугового разряда / Под ред. М.Ф. Жукова. Институт теплофизики CO AH CCCP, 1990. C. 268.

[18] Parker J.V. // MEGAGAUSS-V. Ed. Titov V.M., Shevtsov G.A. Nova Publishers. NY. 1990. P. 781-788.

[19] Witherspoon F.D., Burton R.L., Goldsmith S.A. // IEEE Trans. Magnetics. 1989. Vol. 25. N 1. P. 609-614. DOI: $10.1109 / 20.22609$

[20] Harry D. Fair, Electric Launch Science and Technology in the United States Труды Международного Семинара „Гидродинамика высоких плотностей энергии“ / Под ред. Г.А. Швецова. Новосибирск: Изд-во Института гидродинамики СО РАН, 2004. С. 256-266.

[21] Дробышевский Э.М., Жуков Б.Г., Назаров Е.В., Розов С.И., Соколов В.М., Куракин Р.О., Савельев М.А. // ЖТФ. 1991. T. 61. № 4. С. 170-179.

[22] Райзер Ю.П. Физика газового разряда. М.: Наука, 1987. $591 \mathrm{c}$

[23] Физические величины. Справочник / Под ред. И.С. Григорьева, Е.3. Мейлихова. М.: Энергоатомиздат, 1991. 1260 с.

[24] Kozlov A.V., Polistchook V.P., Shurupov A.V., Shurupova N.P. // XVI ${ }^{\text {th }}$ International Symposium on Electromagnetic Launch Technology, China. Beijing. 15-19 May. 2012. DOI: 10.1109/EML.2012.6325113

[25] Тимошенко С.П., Янг Д.Х., Уивер У. Колебания в инженерном деле. М.: Машиностроение, 1985. 470 с.

[26] Jones J.P., Bhuta P.G. // J. Appl. Mechanics. Transactions of ASME. 1964. Vol. 31. P. 105-111.

[27] Lewis K.B., Nechitailo N.V. // IEEE Transactions on Magnetics. 2007. Vol. 43. N 1. P. 157-162. DOI: 10.1109/TMAG.2006.887648

[28] Upshaw J.L., Zowarka R.C., Cook R.W., Weeks D.A. // IEEE Trans. on Magnetics. 1989. Vol. 25. N 1. P. 568-573.

[29] Козлов А.В., Котов А.В., Полишук В.П., Шурупов А.В. // Вестник ОИВТ РАН. 2018. № 1. С. 22-26.

[30] Simkins T.E. // J. Sound and Vibration. 1994. Vol. 172. N 2. P. $145-154$. 